\title{
Distributed Networked Set-Membership Filtering with Ellipsoidal State Estimations
}

\author{
Nan Xia ${ }^{\mathrm{a}}$, Fuwen Yang ${ }^{\mathrm{b}, *}$, Qing-Long $\mathrm{Han}^{\mathrm{c}, *}$ \\ ${ }^{a}$ School of Information Science and Engineering, East China University of Science and \\ Technology, 130 Meilong Road, Shanghai, 200237, China \\ ${ }^{b}$ Griffith School of Engineering, Griffith University, Gold Coast Campus, Qld 4222, \\ Australia \\ ${ }^{c}$ School of Software and Electrical Engineering, Swinburne University of Technology, \\ Hawthorn, Melbourne, VIC 3122, Australia

\section{Abstract}

This paper addresses the problem of distributed networked set-membership filtering with ellipsoidal state estimations for a class of discrete time-varying systems in the presence of unknown-but-bounded process and measurement noises. Both global and local ellipsoidal state estimations are provided to locate the true state (target) via a distributed filtering network. A new geometric method based on Minkowski sum is proposed to produce the global ellipsoidal estimation. A novel convex optimization approach is developed to derive some sufficient conditions on the existence of local networked set-membership filters and to obtain the local ellipsoidal estimations by exchanging information among neighboring filters via communication networks. An experiment is conducted based on a 2-kW single-phase grid-connected power generation system platform to demonstrate the feasibility and the effectiveness of the proposed method in the real application.

Keywords: Distributed networked set-membership filtering, ellipsoidal state estimation, geometric method, unknown-but-bounded noise, convex optimization approach.

\section{1}

${ }^{*}$ Corresponding authors: Fuwen Yang, E-mail: fuwen.yang@griffith.edu.au; Qing-Long Han, Email: qhan@swin.edu.au 


\section{Introduction}

Recently, the distributed filtering (or estimation) technology has become a promising research topic. This technology offers a number of filters in a distributed manner to independently estimate the state of a plant that resides in its connection (or communication) ranges and to share the estimation cooperatively by exchanging information with neighbors. This feature enables the distributed filtering to present great advantages, such as separating the computational load and facilitating fault detection, over the centralized filtering relying on a fusion to deal with all the measurements [4, 9]. Therefore, the distributed filtering has been widely applied in networked systems [15, 23, 42, 40, 29, 28, 13, 41], networked monitoring systems [5, 7, 24, 35, 18], multi-agent consensus $[2,20,6,25,14,12]$, and autonomous navigation $[22,30]$, and so on.

In the existing results, there are mainly two approaches for the distributed filtering, i.e., the distributed Kalman filtering (DKF) $[2,3,9,19,21]$ and the distributed $H_{\infty}$ filtering (DHF) $[38,5,8,10,11,25,27,36]$. The DKF has collaborative information processing by Kalman filters in a distributed manner where each filter collects information around its neighbors connected by a communication network. However, the DKF is restrictive in application because it requires very detailed statistical information of process and measurement noises, and it may not be robust against plant uncertainty. Alternatively, the DHF usually safeguards against the worst case of disturbances and thus does not require knowledge of statistical properties for noises. It can minimize a bound on the variance of the estimation error over all possible disturbances while satisfying a certain prescribed $H_{\infty}$ performance. Therefore, it is over-conservative and results in a better robust behavior to disturbance variation. However, both DKF and DHF focus on the optimization of the estimation error and only provide the point-estimation of system states. The region in which the true state resides is not exactly determined. How to locate the true state is crucial for some systems, especially for a tracking system. As a motivation, a set-estimation approach, i.e., the distributed networked set-membership filtering (DNSMF) approach, is proposed in this paper.

As is known, the set-membership filtering (SMF) can provide a set of state estimations in state space, which guarantees to contain the true state of the system in the presence of unknown-but-bounded noises [33, 34]. It is similar 
with the interval algorithm [16, 26]. However, the interval algorithm mainly deals with system parameter uncertainty. Differently, the SMF deals with the uncertainty in system noise based on a determined system model. So far, there are many efficient approaches developed to obtain the solutions to SMF problems [31, 33, 34, 37]. Nevertheless, these approaches are all centralized ones. The distributed ones have not yet received adequate attention. Nowadays, owing to the development of networking technologies, an increasing number of practical systems are connected by networks. Thus the whole estimated system can be more complicated and more geographically dispersed such as the observation stations for locating satellites and rockets, which will naturally form a distributed estimation for locating the targets. Therefore, the problem of DNSMF for target estimation is of significance for practical applications. This is also the motivation for this paper to propose a DNSMF approach. However, there is a vital problem that DNSMF provides a number of local estimation sets of tiny difference due to the influence of different noises in the network. Thus, it is important to develop a new method to integrate all these local estimation sets in a global view, which is the so-called global state estimation method.

In order to obtain the global state estimation, there are two different methods can be used, i.e., the union method and the intersection method. The former is to build a set to contain the union of all the local estimation sets, which provides all possible regions for locating the true state. The latter is to find a set to contain the intersection of all the local estimation sets, which provides the most feasible region for locating the true state. Generally, the intersection method is more popular since it can offer a smaller set to improve the filtering performance, but it has the drawback that there may be even no intersection in case some incorrect measurements or impulse noises appearing. Compared with the intersection method, the union method is more fault-tolerant and reliable. Thus, in this paper, a union method will be adopted to produce the global estimation. And a new geometric method based on Minkowski sum is derived for calculating the global estimation.

In this paper, a DNSMF problem is investigated under unknown-but-bounded process and measurement noises, and some special features of the DNSMF are to be revealed in a distributed way. A global ellipsoidal state estimation is provided via the union method based on Minkowski sum to integrate the local ellipsoidal state estimation sets, which are produced by each local filter in a filtering network to locate the true state. In the filtering network, each local 
filter provides an ellipsoidal state estimation by using local measurement and neighboring information. As a result, both global and local ellipsoidal estimations are provided to locate the true state. The main contributions of this paper can be summarized as follows: (i) A new geometric method based on Minkowski sum is proposed to produce the global ellipsoidal estimation, whose aim is to include the union of all the local ellipsoidal estimations; (ii) The techniques are presented for transforming and expanding the local ellipsoidal estimations to include the origin, which is crucial to determine the global ellipsoidal estimation; and (iii) A time-varying cooperative filtering approach is developed to derive some sufficient conditions for the existence of local set-membership filters by exchanging information among the neighboring filters. A recursive convex optimization algorithm is provided to determine the local ellipsoidal estimations.

The rest of this paper is organized as follows. An intuitive example of the DNSMF problem is formulated in Section 2. Both the global and the local ellipsoidal state estimations are presented in Section 3. Section 4 provides a real experiment to demonstrate the effectiveness of our algorithm. Conclusions and future directions are described in Section 5.

Notation. For symmetric matrices $X$ and $Y$, the notation $X \geq Y$ (respectively, $X>Y$ ) means that $X-Y$ is positive semi-definite (respectively, positive definite). The superscript $T$ stands for matrix transpose. $\operatorname{trace}(P)$ denotes the trace of the matrix $P . \varepsilon(\hat{x}, P)=\left\{x:(x-\hat{x})^{T} P^{-1}(x-\hat{x}) \leq 1, P>0\right\}$ denotes an ellipsoid. 0 means an empty set. $\oplus$ denotes Minkowski sum. $U$ and $\bigcap$ represent the union of sets and the intersection of sets, respectively. $\mathbf{0}=\left[\begin{array}{lll}0 & \cdots & 0\end{array}\right]^{T} \in \mathbb{R}^{n}$

\section{Problem Formulation}

The objective of this paper is to design the distributed networked set-membership filters to estimate the state of an estimated system. Thus, an intuitive example of distributed filtering using $N$ set-membership filters is provided in Fig. 1.

Remark 1. Fig. 1 shows a distributed form of the networked set-membership filters. It consists of $N$ filters and each filter $i$ has a communication domain $\mathbb{N}_{i}$ including its neighboring filters. For large-scale spatial and distributed systems, it is difficult to build a communication network to connect all the filters because a large-scale network may incur high cost and high communication overheads. 


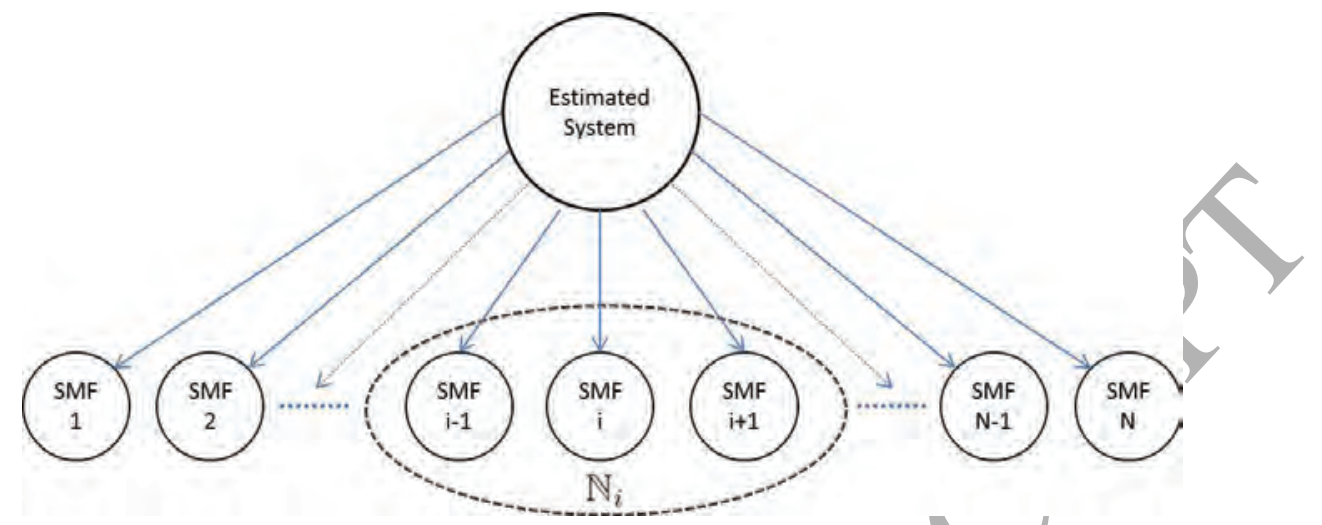

Figure 1: The structure of the distributed networked set-membership filtering

Thus, it is to be established a local communication network to exchange information between the $i_{\text {th }}$ filter and its neighboring filters as the communication domain $\mathbb{N}_{i}$, of which the interconnection topology is shown in Fig. 2. The state information will be collected only by the $i_{\text {th }}$ filter from its neighboring filters in the $\mathbb{N}_{i}$, and the $i_{\text {th }}$ filter will not exchange information among the filters out of $\mathbb{N}_{i}$. For practical interests, other architectures can also be applied for the distributed set-membership filters, such as a circle structure and a star structure.

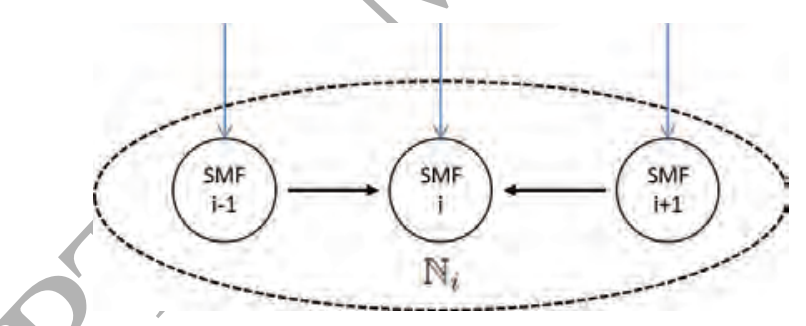

Figure 2: The communication domain $\mathbb{N}_{i}$ of SMF $i$

Consider the following discrete time-varying system as the estimated system in Fig. 1, which is described by

$$
x_{k+1}=F_{k} x_{k}+B_{k} w_{k}
$$

where $x_{k} \in \mathbb{R}^{n}$ is the system state; $F_{k}$ and $B_{k}$ are known time-varying matrices with appropriate dimensions; $w_{k} \in \mathbb{R}^{r}$ is the process noise which is assumed to be unknown but bounded, and to be confined to the following ellipsoid:

$$
w_{k} \in \varepsilon\left(\mathbf{0}, Q_{k}\right)=\left\{w_{k}: w_{k}^{T} Q_{k}^{-1} w_{k} \leq 1\right\} \triangleq W_{k},
$$


where $Q_{k}=Q_{k}^{T}>0$ is a known matrix with compatible dimensions. The initial state $x_{0}$ belongs to a given ellipsoid

$$
x_{0} \in \varepsilon\left(\hat{x}_{0}, P_{0}\right)=\left\{x_{0}:\left(x_{0}-\hat{x}_{0}\right)^{T} P_{0}^{-1}\left(x_{0}-\hat{x}_{0}\right) \leq 1\right\} \triangleq X_{0},
$$

where $\hat{x}_{0}$ is an estimation of $x_{0}$ assumed to be given, and $P_{0}=P_{0}^{T}>0$ is known matrix.

In Fig. 1, each set-membership filter has its own output measurement, which is described by

$$
y_{k}^{i}=C_{k}^{i} x_{k}+D_{k}^{i} v_{k}^{i}, i=1,2, \cdots, N,
$$

where $y_{k}^{i} \in \mathbb{R}^{m}$ is the measurement of the $i_{t h}$ set-membership filter; $C_{k}^{i}$ and $D_{k}^{i}$ are known matrices with appropriate dimensions; $v_{k}^{i} \in \mathbb{R}^{p}$ is the measurement noise which is assumed to be unknown but bounded, and to be confined to the following ellipsoid:

$$
v_{k}^{i} \in \varepsilon\left(\mathbf{0}, R_{k}^{i}\right)=\left\{v_{k}^{i}:\left(v_{k}^{i}\right)^{T}\left(R_{k}^{i}\right)^{-1} v_{k}^{i} \leq 1\right\} \triangleq V_{k}^{i}, i=1, \cdots, N,
$$

where $R_{k}^{i}=\left(R_{k}^{i}\right)^{T}>0$ is a known matrix with compatible dimensions.

In DNSMF, each filter not only adopts its local measured information, but also takes the state information from its neighbors. The communication domain $\mathbb{N}_{i}$ contains the $n_{i}$ filters that provide information for the set-membership filter $i$, see Fig. 1. The filter $i$ estimates the state $x_{k}$, which is governed by

$$
\hat{x}_{k \neq 1}^{i}=G_{k}^{i} \hat{x}_{k}^{i}+L_{k}^{i} y_{k+1}^{i}+K_{k}^{i} \sum_{j \in \mathbb{N}_{i}} a_{i j}\left(\hat{x}_{k}^{i j}-\hat{x}_{k}^{i}\right),
$$

where $\hat{x}_{k}^{i} \in \mathbb{R}^{n}$ is the estimation of the state $x_{k}$ provided by the filter $i ; \hat{x}_{k}^{i j} \in \mathbb{R}^{n}$ is the estimation of the state $x_{k}$ provided by the neighboring filter $j$ in $\mathbb{N}_{i}$, where $j=1, \cdots, n_{i} ; G_{k}^{i}, L_{k}^{i}$ and $K_{k}^{i}$ are the filter parameters to be determined; $a_{i j} \geq 0$ is the weighting coefficient that represents the influence of the filter $j$ 's neighboring estimation on the filter $i$ 's estimation. If $j=i$, then $a_{i j}=0$. Therefore, each filter provides the state estimation which relies on its local measurement $y_{k+1}^{i}{ }_{1}$ and its neighboring estimations $\hat{x}_{k}^{i j}$ s received from its neighbors. Then, the obtained estimation is also transmitted to the neighbors for use.

In DNSMF, each set-membership filter provides an ellipsoidal state estimation by using its own information and its neighboring information. Due to different measurements and unknown noises, each local filter provides a different ellipsoidal estimation, and the true state (the target) may locate anywhere 
in each ellipsoid. For ensuring the reliability of the estimation result, a global ellipsoidal state estimation will be derived for including the union of all local estimations. As a result, the true states can be determined by the global estimation. For this purpose, the objective of this paper is to provide:

i) Local ellipsoidal state estimation: Compute an ellipsoid in which the timated state resides based on the local set-membership filter, i.e., compute an ellipsoid $X_{k}^{i}$ such that

$$
X_{k}^{i}=\varepsilon\left(\hat{x}_{k}^{i}, P_{k}^{i}\right)=\left\{x_{k} \in \mathbb{R}^{n} \mid\left(x_{k}-\hat{x}_{k}^{i}\right)^{T}\left(P_{k}^{i}\right)^{-1}\left(x_{k}-\hat{x}_{k}^{i}\right) \leq 1\right\}
$$

ii) Global ellipsoidal state estimation: Compute a global ellipsoid containing the union of all local estimated ellipsoids, i.e., compute an ellipsoid $X_{k}$ such that

$$
X_{k}=\varepsilon\left(\hat{x}_{k}, P_{k}\right) \supseteq X_{k}^{1} \bigcup \cdots \bigcup X_{k}^{i} \bigcup \cdots \bigcup X_{k}^{N},
$$

where $X_{k}^{i}$ is the $i_{t h}$ local ellipsoidal state estimation.

\section{Ellipsoidal State Estimation}

In this section, an ellipsoid $X_{k}$ will be derived in which the estimated state resides. Both global and local ellipsoidal state estimations are presented. A novel geometric method based on Minkowski sum will be proposed to compute the global ellipsoidal estimation to integrate the local ellipsoidal state estimations. The local estimations $X_{k}^{i}$ s will be produced to satisfy the optimal criterion of an ellipsoid, and a convex optimization recursive algorithm will be provided for designing the local set-membership filters.

\subsection{Global Ellipsoidal State Estimation}

The global ellipsoidal state estimation is designed to cover the union of all the local estimation ellipsoids. In this sub-section, a geometric method is proposed to compute the global ellipsoidal state estimation based on Minkowski sum. In order to perform the global estimation, the definition of Minkowski sum and the minimum external ellipsoid of Minkowski sum are introduced as follows.

Definition 1. [17] Consider $m$ ellipsoids $\varepsilon_{i}=\varepsilon\left(a_{i}, Q_{i}\right), i=1, \cdots, m$, where $a_{i} \in \mathbb{R}^{n}, Q_{i} \in \ell\left(\mathbb{R}^{n}, \mathbb{R}^{n}\right)$ and $Q_{i}>0$. Then, their Minkowski sum $\varepsilon_{s}$ is defined $a s$

$\varepsilon_{s}=\varepsilon_{1} \oplus \cdots \oplus \varepsilon_{i} \oplus \cdots \oplus \varepsilon_{m}=\left\{\left(e_{1}+\cdots+e_{i}+\cdots+e_{m}\right) \mid e_{1} \in \varepsilon_{1}, \cdots, e_{i} \in \varepsilon_{i}, \cdots, e_{m} \in \varepsilon_{m}\right\}$. 
Lemma 1. [17] The minimum external ellipsoid $\varepsilon_{m i n}$ of the Minkowski sum $\varepsilon_{s}$ of $m$ ellipsoids $\varepsilon_{i}=\varepsilon\left(a_{i}, Q_{i}\right)$ is determined by

$$
\varepsilon_{\min }=\varepsilon(a, Q)
$$

where

$$
a=\sum_{i=1}^{m} a_{i}
$$

and

$$
Q=\sum_{i=1}^{m} p_{i} \cdot\left(\sum_{i=1}^{m} p_{i}^{-1} Q_{i}\right)
$$

with $p_{i}=\sqrt{\operatorname{trace}\left(Q_{i}\right)}, i=1, \cdots, m$. Therefore,

$$
\varepsilon_{\text {min }} \supseteq \varepsilon_{s}=\varepsilon_{1} \oplus \cdots \oplus \varepsilon_{i} \oplus \cdots \oplus \varepsilon_{m} .
$$

Remark 2. Definition 1 gives a solution to form the geometric sum of local estimations, and the Lemma 1 is used to produce the final global ellipsoidal estimation. To some extent, Minkowski sum can be directly utilized to solve the problem of the geometric sum of several convex sets. But Minkowski sum is essentially the sum of vectors represented by those points in subsets, see Definition 1. As a result, the sum may not exactly the union of all the subsets. That is reason for generating a novel geometric algorithm based on Minkowski sum rather than directly applying Minkowski sum to find a set that includes the union of all the local estimation ellipsoids in our problem.

Before obtaining the set to cover the union of all the sets of the local estimation ellipsoids, the following theorem is firstly introduced to tell the feature of the union.

Theorem 1. Suppose that there are two ellipsoids $\varepsilon_{1}=\varepsilon\left(a_{1}, Q_{1}\right)$ and $\varepsilon_{2}=$ $\varepsilon\left(a_{2}, Q_{2}\right)$. If there exists an external ellipsoid $\varepsilon_{e}$ such that the Minkowski sum $\varepsilon_{s}$ of $\varepsilon_{1}$ and $\sigma_{2}$ is included in $\varepsilon_{e}$, i.e., $\varepsilon_{e} \supseteq \varepsilon_{s}=\varepsilon_{1} \oplus \varepsilon_{2}$, then we have the following statements:

i) If $\boldsymbol{O} \in \varepsilon_{1}$, then $\varepsilon_{2} \subseteq \varepsilon_{e}$.

ii) If $\boldsymbol{O} \in \varepsilon_{1} \bigcap \varepsilon_{2}$, then $\varepsilon_{1} \bigcup \varepsilon_{2} \subseteq \varepsilon_{e}$.

Proof: i) Obviously, when $\mathbf{0} \in \varepsilon_{1}, \varepsilon_{s}=\varepsilon_{1} \oplus \varepsilon_{2}$ can be rewritten as

$$
\begin{aligned}
\varepsilon_{s} & =\left\{\left(\mathbf{0}+e_{2}\right) \mid e_{2} \in \varepsilon_{2}\right\} \bigcup\left\{\left(e_{1}+e_{2}\right) \mid e_{1} \in \varepsilon_{1}, e_{1} \neq \mathbf{0}, e_{2} \in \varepsilon_{2}\right\} \\
& =\varepsilon_{2} \bigcup\left\{\left(e_{1}+e_{2}\right) \mid e_{1} \in \varepsilon_{1}, e_{1} \neq \mathbf{0}, e_{2} \in \varepsilon_{2}\right\} .
\end{aligned}
$$


Since $\varepsilon_{e} \supseteq \varepsilon_{s}=\varepsilon_{1} \oplus \varepsilon_{2}$, there is $\varepsilon_{2} \subseteq \varepsilon_{e}$.

ii) Based on the result of i), we have

$$
\mathbf{0} \in \varepsilon_{1} \bigcap \varepsilon_{2} \Rightarrow\left\{\begin{array}{c}
\mathbf{0} \in \varepsilon_{1} \Rightarrow \varepsilon_{2} \subseteq \varepsilon_{e} \\
\mathbf{0} \in \varepsilon_{2} \Rightarrow \varepsilon_{1} \subseteq \varepsilon_{e}
\end{array}\right\} \Rightarrow \varepsilon_{1} \bigcup \varepsilon_{2} \subseteq \varepsilon_{e},
$$

which ends the proof.

Remark 3. Theorem 1 reveals the important feature of the union of two ellipsoids. If two ellipsoids $\varepsilon_{1}$ and $\varepsilon_{2}$ both contain the origin, their union will be included in an external ellipsoid $\varepsilon_{e}$ of their Minkowski sum $\varepsilon_{s}$. Therefore, an external ellipsoid can be found through this feature to cover the union of the local ellipsoids $\varepsilon_{i}$. And without loss of generality, the minimal external ellipsoid $\varepsilon_{\text {min }}$ is chosen as the external ellipsoid in the following steps, which can be easily calculated by Lemma 1.

In order to clearly explain the feature described in Theorem 1 , an example is provided in Fig. 3. In Fig. 3(a), $\varepsilon_{1}=\varepsilon\left(a_{1}, Q_{1}\right)$, where $a_{1}=\left[\begin{array}{ll}1, & 2\end{array}\right]^{T}$ and $Q_{1}=$ $\left[\begin{array}{cc}10 & 0 \\ 0 & 10\end{array}\right]$, and $\varepsilon_{2}=\varepsilon\left(a_{2}, Q_{2}\right)$, where $a_{2}=\left[\begin{array}{ll}7, & 8\end{array}\right]^{T}$ and $Q_{2}=\left[\begin{array}{cc}10 & 0 \\ 0 & 10\end{array}\right]$. It is clear that $\mathbf{0} \in \varepsilon_{1}$ and thus $\varepsilon_{2}$ is included in the external ellipsoid $\varepsilon_{\min }$ of the Minkovski sum of $\varepsilon_{1}$ and $\varepsilon_{2}$, i.e., $\varepsilon_{2} \subseteq \varepsilon_{\min }$. In Fig. 3(b), $\varepsilon_{1}=\varepsilon\left(a_{1}, Q_{1}\right)$, where $a_{1}=\left[\begin{array}{ll}1, & 2\end{array}\right]^{T}$ and $Q_{1}=\left[\begin{array}{cc}10 & 0 \\ 0 & 10\end{array}\right]$, and $\varepsilon_{2}=\varepsilon\left(a_{2}, Q_{2}\right)$, where $a_{2}=\left[\begin{array}{ll}0.5, & 0.5\end{array}\right]^{T}$ and $Q_{2}=\left[\begin{array}{ll}2 & 0 \\ 0 & 2\end{array}\right]$. It is clear that $\mathbf{0} \in \varepsilon_{1} \cap \varepsilon_{2}$ and therefore $\varepsilon_{1} \bigcup \varepsilon_{2} \subseteq \varepsilon_{\text {min }}$.

Remark 4. Fig. 3 gives an illustrative example to reveal the feature described in Theorèm 1, which also indicates that Minkovski sum cannot make sure to cover the union of local ellipsoids. Moreover, Fig. 3 also reveals that the including of the origin is the key point of the proposed algorithm for the reliability of the global estimation.

Inspired by Theorem 1, the following theorem can be obtained as an expansion from two ellipsoids to $m$ ellipsoids.

Theorem 2. Consider m ellipsoids $\varepsilon_{i}=\varepsilon\left(a_{i}, Q_{i}\right), i=1, \cdots, m$. If $\boldsymbol{O} \in \varepsilon_{1} \bigcap \cdots \bigcap \varepsilon_{m}$, then $\varepsilon_{1} \bigcup \cdots \bigcup \varepsilon_{m} \subseteq \varepsilon_{\text {min }}$, where $\varepsilon_{\text {min }}$ is the minimum external ellipsoid of the Minkowski sum $\varepsilon_{s}$ defined by $\varepsilon_{s}=\varepsilon_{1} \oplus \varepsilon_{2} \oplus \cdots \oplus \varepsilon_{m}$. 


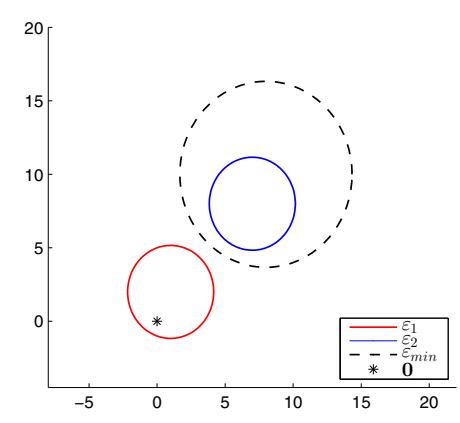

(a)

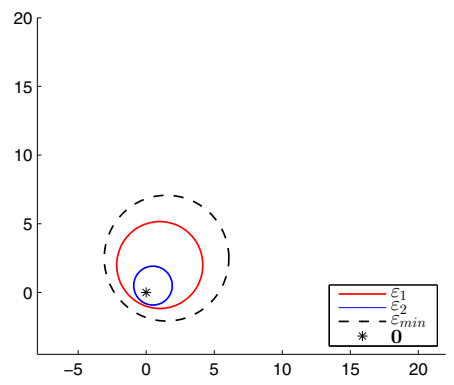

(b)

Figure 3: Examples of two ellipsoids and the minimum external ellipsoid of their Minkowski sum

Proof: From Definition 1, since $\mathbf{0} \in \varepsilon_{1} \bigcap \cdots \varepsilon_{m}$, the Minkowski sum $\varepsilon_{s}=\varepsilon_{1} \oplus \varepsilon_{2} \oplus \cdots \oplus \varepsilon_{m}$ can be rewritten as

$$
\begin{aligned}
\varepsilon_{s}= & \left\{\left(e_{1}+\mathbf{0}+\cdots+\mathbf{0}\right) \mid e_{1} \in \varepsilon_{1}\right\} \bigcup\left\{\left(e_{1}+\cdots+e_{m}\right) \mid e_{i} \in \varepsilon_{i}, i=1,2, \cdots, m, e_{j} \neq \mathbf{0},\right. \\
& j=2,3, \cdots, m\} \bigcup\left\{\left(\mathbf{0}+e_{2}+\mathbf{0} \cdots+\mathbf{0}\right) \mid e_{2} \in \varepsilon_{2}\right\} \bigcup\left\{\left(e_{1}+\cdots+e_{m}\right) \mid e_{i} \in \varepsilon_{i},\right. \\
& \left.i=1,2, \cdots, m, e_{j} \neq \mathbf{0}, j=1,3, \cdots, m\right\} \bigcup \cdots \bigcup\left\{\left(\mathbf{0}+\cdots+\mathbf{0}+e_{m}\right) \mid e_{m} \in \varepsilon_{m}\right\} \\
& \bigcup\left\{\left(e_{1}+\cdots+e_{m}\right) \mid e_{i} \in \varepsilon_{i}, i=1,2, \cdots, m, e_{j} \neq \mathbf{0}, j=1,2, \cdots, m-1\right\} \\
= & \varepsilon_{1} \bigcup \varepsilon_{2} \bigcup \cdots \bigcup \varepsilon_{m} \bigcup\left\{\left(e_{1}+\cdots+e_{m}\right) \mid e_{i} \in \varepsilon_{i}, i=1,2, \cdots, m, e_{j} \neq \mathbf{0}, j=2,3, \cdots, m\right\} \\
& \bigcup\left\{\left(e_{1}+\cdots+e_{m}\right) \mid e_{i} \in \varepsilon_{i}, i=1,2, \cdots, m, e_{j} \neq \mathbf{0}, j=1,3, \cdots, m\right\} \bigcup \cdots \\
& \bigcup\left\{\left(e_{1}+\cdots+e_{m}\right) \mid e_{i} \in \varepsilon_{i}, i=1,2, \cdots, m, e_{j} \neq \mathbf{0}, j=1,2, \cdots, m-1\right\},
\end{aligned}
$$

which implies that $\varepsilon_{1} \bigcup \varepsilon_{2} \bigcup \cdots \cup \varepsilon_{m} \subseteq \varepsilon_{s}$. Through Lemma 1, there is

$$
\varepsilon_{1} \bigcup \varepsilon_{2} \bigcup \cdots \bigcup \varepsilon_{m} \subseteq \varepsilon_{m i n}
$$

which ends the proof.

Remark 5. Theorem 2 provides an expanded solution of Theorem 1 that the union of $m$ ellipsoids can be included in the minimal external ellipsoid of their Minkowski sum. To sum up, the union of all the ellipsoids $\left\{\varepsilon_{1}, \cdots, \varepsilon_{m}\right\}$ can be included in the minimal external ellipsoid $\varepsilon_{\text {min }}$ of their Minkowski sum $\varepsilon_{s}$ if they all contain the origin.

We now in a position to derive the global ellipsoidal estimation $X_{k}$. Suppose that the local ellipsoidal estimations are described as $X_{k}^{i}=\varepsilon\left(\hat{x}_{k}^{i}, P_{k}^{i}\right)$, where $\hat{x}_{k}^{i}$ 
is the estimated center and $P_{k}^{i}$ is its corresponding shape matrix at instant $k$, $i=1,2, \cdots, N$. Firstly, one needs to obtain a group of new ellipsoids $\bar{X}_{k}^{i}$ s via the translation of the initial estimations $X_{k}^{i} \mathrm{~s}$ in position to satisfy the requirements that a) the mean value of the centers of $\bar{X}_{k}^{i}$ s is zero; b) the relative position of $\bar{X}_{k}^{i}$ s among each other is the same as that of the initial estimations $X_{k}^{i}$ s. That is to say, the $\bar{X}_{k}^{i}$ is obtained by transforming the $X_{k}^{i}$ as

$$
\bar{X}_{k}^{i}=\varepsilon\left(\bar{x}_{k}^{i}, P_{k}^{i}\right)=\varepsilon\left(\hat{x}_{k}^{i}-\bar{x}_{k}, P_{k}^{i}\right), i=1,2, \cdots, N,
$$

where

$$
\bar{x}_{k}=\frac{1}{N} \sum_{i=1}^{N} \hat{x}_{k}^{i} .
$$

Secondly, one needs to judge whether each $\bar{X}_{k}^{i}$ includes the origin, i.e., $\mathbf{0} \in$ $\bar{X}_{k}^{i}$. If $\mathbf{0} \in \bar{X}_{k}^{i}$, the $\bar{X}_{k}^{i}$ can be used to build the global estimation directly in the next steps. Once $\mathbf{0} \notin \bar{X}_{k}^{i}$, another new ellipsoid $S_{k}^{i}$ will be constructed to include both $\bar{X}_{k}^{i}$ and the origin, of which the construction is as follows.

In order to construct a $S_{k}^{i}$, an ellipsoid $\Theta_{k}^{i}=\varepsilon\left(\mathbf{0}, Q_{k}^{i}\right)$ centered at the origin needs to be produced, which should be intersected with $\bar{X}_{k}^{i}$. Thus, $\Theta_{k}^{i}$ has the following properties:

i) There is a point $a \in \bar{X}_{k}^{i} \cap \Theta_{k}^{i}$, i.e., $\bar{X}_{k}^{i} \cap \Theta_{k}^{i} \neq \emptyset$.

ii) There is a point $b \in \Theta_{k}^{i}$ such that $a+b=\mathbf{0}$, i.e., $b$ is the symmetric point of $a$.

Based on Definition 1, we have

$$
\begin{aligned}
\bar{X}_{k}^{i} \oplus \Theta_{k}^{i}= & \{a+b\} \bigcup\left\{\left(\bar{x}^{i}+\theta^{i}\right) \mid \bar{x}^{i} \in \bar{X}_{k}^{i}, \bar{x}^{i} \neq a, \theta^{i} \in \Theta_{k}^{i}\right\} \\
& \bigcup\left\{\left(\bar{x}^{i}+\theta^{i}\right) \mid \bar{x}^{i} \in \bar{X}_{k}^{i}, \theta^{i} \in \Theta_{k}^{i}, \theta^{i} \neq b\right\} \\
= & \{\mathbf{0}\} \bigcup\left\{\left(\bar{x}^{i}+\theta^{i}\right) \mid \bar{x}^{i} \in \bar{X}_{k}^{i}, \bar{x}^{i} \neq a, \theta^{i} \in \Theta_{k}^{i}\right\} \\
& \bigcup\left\{\left(\bar{x}^{i}+\theta^{i}\right) \mid \bar{x}^{i} \in \bar{X}_{k}^{i}, \theta^{i} \in \Theta_{k}^{i}, \theta^{i} \neq b\right\} .
\end{aligned}
$$

it is clear that $\mathbf{0} \in \bar{X}_{k}^{i} \oplus \Theta_{k}^{i}$.

After producing an ellipsoid $\Theta_{k}^{i}=\varepsilon\left(\mathbf{0}, Q_{k}^{i}\right)$, one still needs to minimize the $\Theta_{k}^{i}$ to make it tangential to the $\bar{X}_{k}^{i}$. Therefore, the following optimization method is proposed to generate the minimal ellipsoid $\Theta_{k}^{i}$.

$$
\begin{aligned}
& \min \operatorname{trace}\left(Q_{k}^{i}\right) \text {. } \\
& \text { s.t. } \quad Q_{k}^{i}>0 \\
& \bar{X}_{k}^{i} \cap \Theta_{k}^{i} \neq \emptyset
\end{aligned}
$$


When $\Theta_{k}^{i}$ is generated, the sum $\bar{X}_{k}^{i} \oplus \Theta_{k}^{i}$ is obtained. According to Lemma 1, the minimum external ellipsoid of the Minkowski sum of $\bar{X}_{k}^{i}$ and $\Theta_{k}^{i}$ is obtained by $\varepsilon\left(s_{k}^{i}, M_{k}^{i}\right)$, where

$$
s_{k}^{i}=\bar{x}_{k}^{i}+\mathbf{0}=\bar{x}_{k}^{i}
$$

and

$$
M_{k}^{i}=\left(p_{1}+p_{2}\right)\left(p_{1}^{-1} P_{k}^{i}+p_{2}^{-1} Q_{k}^{i}\right)
$$

with $p_{1}=\sqrt{\operatorname{trace}\left(P_{k}^{i}\right)}$ and $p_{2}=\sqrt{\operatorname{trace}\left(Q_{k}^{i}\right)}$. Then, $\mathbf{0} \in \bar{X}_{k}^{i} \oplus \Theta_{k}^{i} \subseteq \varepsilon\left(s_{k}^{i}, M_{k}^{i}\right)$. Thus, for the convenience of description, the form of $S_{k}^{i}$ can be summarized as

$$
S_{k}^{i}=\varepsilon\left(\bar{s}_{k}^{i}, \bar{P}_{k}^{i}\right)=\left\{\begin{array}{cl}
\bar{X}_{k}^{i} & , \quad \mathbf{0} \in \bar{X}_{k}^{i}, \\
\varepsilon\left(s_{k}^{i}, M_{k}^{i}\right) & , \quad \mathbf{0} \notin \bar{X}_{k}^{i},
\end{array}\right.
$$

where the $\bar{s}_{k}^{i}$ and the $\bar{P}_{k}^{i}$ are the center and shape matrix of the $S_{k}^{i}$ respectively. According to Theorem 1 and (9), it is obvious that $\bar{X}_{k}^{i} \bigcup\{0\} \subseteq S_{k}^{i}$.

Remark 6. The second step is to form the ellipsoid $S_{k}^{i}$ to contain both the origin and $\bar{X}_{k}^{i}$ in order to apply Theorem 2 for the reliability of producing the global estimation. The inclusion of the $\bar{X}_{k}^{i}$ is equal to the inclusion of the true state, and the inclusion of the origin can help to apply Theorem 2. Thus, both the $\bar{X}_{k}^{i}$ and the origin are significant to be covered in this step.

Thirdly, an ellipsoid $\bar{S}_{k}$ is to be found to enclose all the $S_{k}^{i}, i=1,2, \cdots, N$. According to Lemma $1, S_{k}$ can be calculated as

$$
\bar{S}_{k}=\varepsilon\left(\bar{s}_{k}, \bar{P}_{k}\right)
$$

where

$$
\bar{s}_{k}=\sum_{i=1}^{N} \bar{s}_{k}^{i}
$$

and

$$
\bar{P}_{k}=\sum_{i=1}^{N} \bar{p}_{k}^{i} \cdot\left(\sum_{i=1}^{N}\left(\bar{p}_{k}^{i}\right)^{-1} \bar{P}_{k}^{i}\right)
$$

with $\bar{p}_{k}^{i}=\sqrt{\operatorname{trace}\left(\bar{P}_{k}^{i}\right)}, i=1, \cdots, N$. Therefore,

$$
\bar{S}_{k}=\varepsilon\left(\bar{s}_{k}, \bar{P}_{k}\right) \supseteq S_{k}^{1} \oplus S_{k}^{2} \oplus \cdots \oplus S_{k}^{N} .
$$

Owing to $\mathbf{0} \in S_{k}^{1} \cap \cdots \bigcap S_{k}^{N}$ and Theorem 2, it is obvious that

$$
\bar{S}_{k} \supseteq S_{k}^{1} \bigcup \cdots \bigcup S_{k}^{N}
$$


Finally, through the translation in position again, the $\bar{S}_{k}$ should be transformed back to the original mean point of the centers of the original local estimations, i.e.,

$$
X_{k}=\varepsilon\left(\hat{x}_{k}, P_{k}\right)=\varepsilon\left(\bar{s}_{k}+\bar{x}_{k}, \bar{P}_{k}\right) .
$$

Thus, the $X_{k}$ in (7) is obtained.

In summary, the following procedures are formed to compute the global ellipsoidal state estimation (Global Estimation Algorithm):

i) Transform the local ellipsoidal state estimations $X_{k}^{i}$ s into $\bar{X}_{k}^{i} \mathrm{~s}$ by (8).

ii) Generate the expanded ellipsoids $S_{k}^{i} \mathrm{~s}$ by $(9)$.

iii) Produce the ellipsoid $\bar{S}_{k}$ for covering the union of all the expanded ellipsoids $S_{k}^{i}$ s by $(10)$.

iv) Transform $\bar{S}_{k}$ to obtain the global ellipsoidal estimation $X_{k}$ by (11).

Remark 7. The whole procedure of the global estimation algorithm is operating on the transformation of the local estimation sets. Note that if the generated global ellipsoid can cover these transformed local estimations, it can definitely contain the union of initial local estimations after translation. Thus, the first step is to transform the initial local estimations by translation towards the origin for the convenience of the calculation. The second and the third steps are to generate the global ellipsoidal set over these transformed ellipsoids based on Theorem 1 and 2. The final step is to return the produced global ellipsoid over the initial local estimations by translation, which can obviously provide the required global estimation set.

Therefore, the global ellipsoidal state estimation can be obtained as long as all the local estimations $X_{k}^{i}$ s are known. In the following sub-section, the local ellipsoidal state estimation will be performed.

\subsection{Local Ellipsoidal State Estimation}

In this subsection, we provide a convex optimization recursive algorithm for computing the local state estimation ellipsoids $X_{k}^{i}$ s for DNSMF. The conditions on the existence of the local ellipsoidal estimation $X_{k}^{i}$ are derived in the following theorem satisfying the optimal criterion of an ellipsoid in which the estimated state resides.

Theorem 3. For the system (1)-(3), suppose that the state $x_{k}$ belongs to its state estimation ellipsoid $\varepsilon\left(\hat{x}_{k}^{i}, P_{k}^{i}\right)$. Then, the one-step ahead state $x_{k+1}$ resides 
in its ellipsoidal state estimation $\varepsilon\left(\hat{x}_{k+1}^{i}, P_{k+1}^{i}\right)$ if there exist matrices $P_{k+1}^{i}>0$, $G_{k}^{i}, L_{k}^{i}, K_{k}^{i}$, and positive scalars $\tau_{1}^{i}, \tau_{2}^{i}$ and $\tau_{3}^{i}$ such that

$$
\left[\begin{array}{cc}
-P_{k+1}^{i} & \Phi_{k}^{i} \\
\Phi_{k}^{i T} & -\Psi\left(\tau_{1}^{i}, \tau_{2}^{i}, \tau_{3}^{i}\right)
\end{array}\right] \leq 0
$$

where

$$
\begin{aligned}
& \Phi_{k}^{i}=\left[\left(I-L_{k}^{i} C_{k+1}^{i}\right) F_{k} \hat{x}_{k}^{i}-G_{k}^{i} \hat{x}_{k}^{i}-K_{k}^{i} \sum_{j \in \mathbb{N}_{i}} a_{i j}\left(\hat{x}_{k}^{i j}-\hat{x}_{k}^{i}\right),\left(I-L_{k}^{i} C_{k+1}^{i}\right) F_{k} E_{k}^{i},\right. \\
& \left.\quad\left(I-L_{k}^{i} C_{k+1}^{i}\right) B_{k},-L_{k}^{i} D_{k+1}^{i}\right], \\
& \Psi\left(\tau_{1}^{i}, \tau_{2}^{i}, \tau_{3}^{i}\right)=\operatorname{diag}\left\{\Pi_{k}^{i}, \tau_{1}^{i} I, \tau_{2}^{i} Q_{k}^{-1}, \tau_{3}^{i}\left(R_{k+1}^{i}\right)^{-1}\right\} \\
& \Pi_{k}^{i}=1-\tau_{1}^{i}-\tau_{2}^{i}-\tau_{3}^{i} .
\end{aligned}
$$

Proof: From (1), (3) and (5), we have

$$
\begin{aligned}
x_{k+1}-\hat{x}_{k+1}^{i}= & \left(I-L_{k}^{i} C_{k+1}^{i}\right) F_{k} x_{k}-G_{k}^{i} \hat{x}_{k}^{i}+\left(I-L_{k}^{i} C_{k+1}^{i}\right) B_{k} w_{k} \\
& -K_{k}^{i} \sum_{j \in \mathbb{N}_{i}} a_{i j}\left(\hat{x}_{k}^{i j}-\hat{x}_{k}^{i}\right)-L_{k}^{i} D_{k+1}^{i} v_{k+1}^{i} .
\end{aligned}
$$

If $x_{k}$ resides in $\varepsilon\left(\hat{x}_{k}^{i}, P_{k}^{i}\right)$, there exists $z_{k}^{i}$ with $\left\|z_{k}^{i}\right\| \leq 1$ such that

$$
x_{k}=\hat{x}_{k}^{i}+E_{k}^{i} z_{k}^{i}
$$

where $E_{k}^{i}$ is the factorization of $P_{k}^{i}=E_{k}^{i} E_{k}^{i T}$. Then, for all $j \in \mathbb{N}_{i}$, we have

$$
\begin{aligned}
x_{k+1}-\hat{x}_{k+1}^{i}= & {\left[\left(I-L_{k}^{i} C_{k+1}^{i}\right) F_{k}-G_{k}^{i}\right] \hat{x}_{k}^{i}-K_{k}^{i} \sum_{j \in \mathbb{N}_{i}} a_{i j}\left(\hat{x}_{k}^{i j}-\hat{x}_{k}^{i}\right) } \\
+ & +\left(I-L_{k}^{i} C_{k+1}^{i}\right) F_{k} E_{k}^{i} z_{k}^{i}+\left(I-L_{k}^{i} C_{k+1}^{i}\right) B_{k} w_{k}-L_{k}^{i} D_{k+1}^{i} v_{k+1}^{i} .
\end{aligned}
$$

Denoting $\eta_{k}^{i}=\left[1, z_{k}^{i T}, w_{k}^{T}, v_{k+1}^{i T}\right]^{T}$, we obtain a compact form as

$$
\begin{gathered}
x_{k+1}-\hat{x}_{k+1}^{i}=\Phi_{k}^{i} \eta_{k}^{i} . \\
\text { Hence, }\left(x_{k+1}-\hat{x}_{k+1}^{i}\right)^{T}\left(P_{k+1}^{i}\right)^{-1}\left(x_{k+1}-\hat{x}_{k+1}^{i}\right) \leq 1 \text { can be written as } \\
\eta_{k}^{i T}\left[\Phi_{k}^{i T}\left(P_{k+1}^{i}\right)^{-1} \Phi_{k}^{i}-\operatorname{diag}\left\{1,0_{d_{x}+d_{w}+d_{v}}\right\}\right] \eta_{k}^{i} \leq 0,
\end{gathered}
$$

where $0_{a}$ is a zero matrix with the dimension $a \times a$.

In addition, $\left\|z_{k}^{i}\right\| \leq 1$ can be expressed as

$$
\eta_{k}^{i T} \operatorname{diag}\left\{-1, I, 0_{d_{w}+d_{v}}\right\} \eta_{k}^{i} \leq 0,
$$


and by using (2) and (4), we have

$$
\begin{gathered}
\eta_{k}^{i T} \operatorname{diag}\left\{-1,0_{d_{x}}, Q_{k}^{-1}, 0_{d_{v}}\right\} \eta_{k}^{i} \leq 0, \\
\eta_{k}^{i T} \operatorname{diag}\left\{-1,0_{d_{x}+d_{w}},\left(R_{k+1}^{i}\right)^{-1}\right\} \eta_{k}^{i} \leq 0 .
\end{gathered}
$$

By using an $\mathcal{S}$-procedure, one can see that (13)-(16) hold if there exist positive scalars $\tau_{1}^{i}, \tau_{2}^{i}$ and $\tau_{3}^{i}$ such that

$$
\begin{aligned}
& \Phi_{k}^{i T}\left(P_{k+1}^{i}\right)^{-1} \Phi_{k}^{i}-\operatorname{diag}\left\{1,0_{d_{x}+d_{w}+d_{v}}\right\}-\tau_{1}^{i} \operatorname{diag}\left\{-1, I, 0_{d_{w}+d_{v}}\right\} \\
& -\tau_{2}^{i} \operatorname{diag}\left\{-1,0_{d_{x}}, Q_{k}^{-1}, 0_{d_{v}}\right\}-\tau_{3}^{i} \operatorname{diag}\left\{-1,0_{d_{x}+d_{w}},\left(R_{k+1}^{i}\right)^{-1}\right\} \leq 0,
\end{aligned}
$$

which can be rewritten in a compact form as

$$
\Phi_{k}^{i T}\left(P_{k+1}^{i}\right)^{-1} \Phi_{k}^{i}-\Psi\left(\tau_{1}^{i}, \tau_{2}^{i}, \tau_{3}^{i}\right) \leq 0
$$

By using Schur Complements, (17) is equivalent to (12), which ends the proof.

Theorem 3 provides a method to obtain the local state estimation ellipsoids for DNSMF. In order to find the minimum ellipsoids, a convex optimization approach is derived to determine the optimal ellipsoids. $P_{k+1}^{i}$ are obtained by solving the following optimization problem:

$$
\min _{G_{k}^{i}, L_{k}^{i}, K_{k}^{i}, \tau_{1}^{i}, \pi_{2}^{i}, \tau_{3}^{i}} \operatorname{trace}\left(P_{k+1}^{i}\right),
$$

which is easily solved by the existing semi-definite programming via an interiorpoint algorithm [32]. So far the optimal local ellipsoidal state estimations are obtained, and the global ellipsoidal state estimation can be calculated by (8)(11).

Note that the proposed DNSMF is a recursive approach with solving a feasible LMI problem via an interior-point algorithm, which has a polynomial-time complexity. The row size of the LMI constraint (12) is $\gamma=2 n+r+p+1$ and the number of scalar decision variables is $\alpha=\frac{3}{2} n^{2}+\frac{5}{2} n+3$. Thus, the computational complexity of the proposed approach is proportional to $\gamma \alpha^{3}$. Compared with the DKF approach, which is only a recursive algorithm without LMIs, the proposed approach has more computational complexity. However, the advantage of applying the proposed approach is that it can operate under the unknown-but-bounded noise, which is not suitable for performing the DKF approach. Furthermore, the proposed DNSMF has a good scalability because it is easy to add local filters with expanding the communication domains for the 
information exchange, which is similar to the DKF approach. The performance of each local filter is independent of the total number of local filters. But more neighboring filters in the communication domain $\mathbb{N}_{i}$ indicates better accuracy for the $i_{t h}$ local estimation.

\section{Simulation Results}

In order to verify the effectiveness and to show the implementation of the proposed algorithm on real world datasets, in this section, an experiment is conducted based on a $2-\mathrm{kW}$ single-phase grid-connected power generation system, which parameters are exhibited in Table 1.

Table 1: The parameters of the model system

\begin{tabular}{cc}
\hline DC power source & $360 \mathrm{~V}$ \\
Power & $L: 8 \mathrm{mH} ; \mathrm{C}: 50 \mu \mathrm{F}$ \\
LC filter & $R: 24.2 \Omega ; L: 77 m H ; C: 132 \mu \mathrm{F}$ \\
RLC load & $220 \sqrt{2} \mathrm{~V}$ \\
Grid voltage & $L_{g}: 1 \mathrm{mH} ; R_{g}: 0.1 \Omega$ \\
Grid impedance & $50 H z$ \\
Fundamental frequency & $400 H z$ \\
Sampling frequency & \\
\hline
\end{tabular}

Based on the employed set-up, a discrete-time model system in state space is built to track the state of voltage at the point of common coupling (PCC) of the grid-connected generation system, which is formed as

$$
x_{k+1}=\left[\begin{array}{cc}
\cos (\omega T) & -\sin (\omega T) \\
\sin (\omega T) & \cos (\omega T)
\end{array}\right] x_{k}+\left[\begin{array}{c}
\Delta u \cos (\omega k T+\omega T) \\
\Delta u \sin (\omega k T+\omega T)
\end{array}\right],
$$

where the state $x_{k}=\left[\begin{array}{ll}x_{1 k}, & x_{2 k}\end{array}\right]^{T}, x_{1 k} \in \mathbb{R}$ and $x_{2 k} \in \mathbb{R} ; \omega=100 \pi \mathrm{rad} / \mathrm{s}$ represents the angular fundamental frequency; $k$ is the $k_{t h}$ sampling; $T=2.5 \mathrm{~ms}$ is the sampling period; $\Delta u=10^{-3} U_{0} \sin (\omega k T)$ is the voltage deviation between two sampling intervals, where $U_{0}=220 \sqrt{2} V$ is the voltage amplitude.

In the proposed module of filtering, the designed methods are provided by programming in Matlab, where there are four filters in the designed filtering network. Each filter $i$ will communicate with its two neighboring filters in the domain $\mathbb{N}_{i}$, see Fig. 4. According to the Metropolis weights method ${ }^{1}[1]$, the 


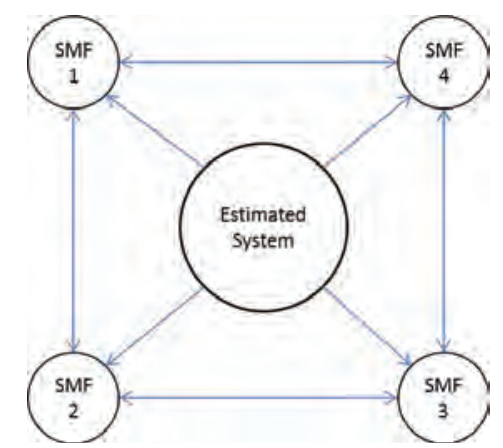

Figure 4: The communication topology graph of the filtering network

weighted matrix of the communication topology is

Then the measurement equation is

$$
\left[a_{i j}\right]=\left[\begin{array}{cccc}
0 & 1 / 3 & 0 & 1 / 3 \\
1 / 3 & 0 & 1 / 3 & 0 \\
0 & 1 / 3 & 0 & 1 / 3 \\
1 / 3 & 0 & 1 / 3 & 0
\end{array}\right] .
$$

$$
y_{k}^{i}=[1+0.1 i, 0] x_{k}+[1+0.1 i] v_{k}^{i},
$$

where $v_{k}^{i}=0.02 U_{0} \sin (\omega k T)(1+0.1 i), i=1,2,3,4$.

$$
i=1,2,3,4 \text {. }
$$

Additionally, $Q_{k}=\left[\begin{array}{cc}\left(0.002 U_{0}\right)^{2} & 0 \\ 0 & \left(0.002 U_{0}\right)^{2}\end{array}\right] ; R_{k}^{i}=\left(0.03 U_{0}\right)^{2}(1+0.1 i)$,

The initial conditions of the states are $x_{0}=[220,220]^{T}, \hat{x}_{0}^{1}=\left[\begin{array}{lll}300.53, & -80.53\end{array}\right]^{T}$, $\hat{x}_{0}^{2}=[295.9,96.14]^{T}, \hat{x}_{0}^{3}=[-182.88,251.71]^{T}, \hat{x}_{0}^{4}=\left[\begin{array}{lll}-287.44, & -119.07\end{array}\right]^{T}$, respectively and $P_{0}^{1}=P_{0}^{2}=P_{0}^{3}=P_{0}^{4}=\left[\begin{array}{cc}4 U_{0}^{2} & 0 \\ 0 & 4 U_{0}^{2}\end{array}\right]$.

The simulation results of the proposed method are obtained under Matlab R2012a from $k=1$ to $k=50$. By solving the optimization problem (18), the results for the phase planes of the position of the true states together with their estimations are depicted in Fig. 5. It is clear that all the true states are included in the estimated ellipsoids, which indicates the good performance of the local state estimations.

\footnotetext{
${ }^{1}$ Metropolis weights: $a_{i j}=1 /\left(1+\max \left[\left|\mathbb{N}_{i}\right|,\left|\mathbb{N}_{j}\right|\right]\right)$
} 
In order to integrate these local estimations, the global ellipsoidal estimation method proposed in Section 3.1 is applied. Fig. 6 illustrates the global state estimation results. Particularly, each global estimation contains the union of all local estimations at each time instant, which also reveals the good performance of the global state estimations.

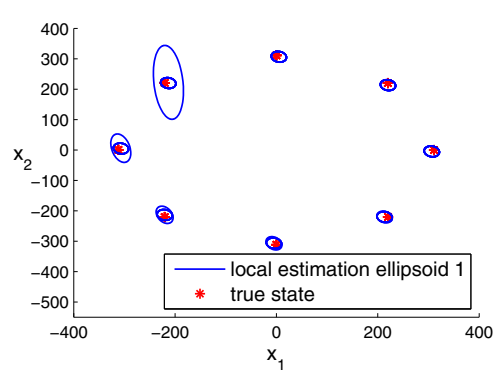

(a)

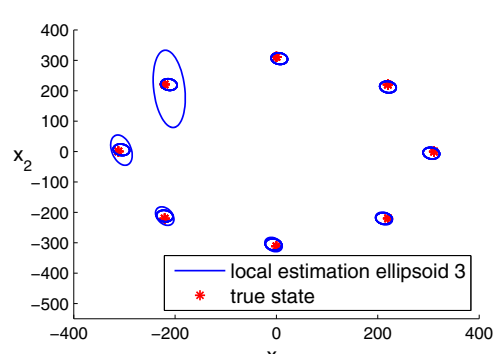

(c)
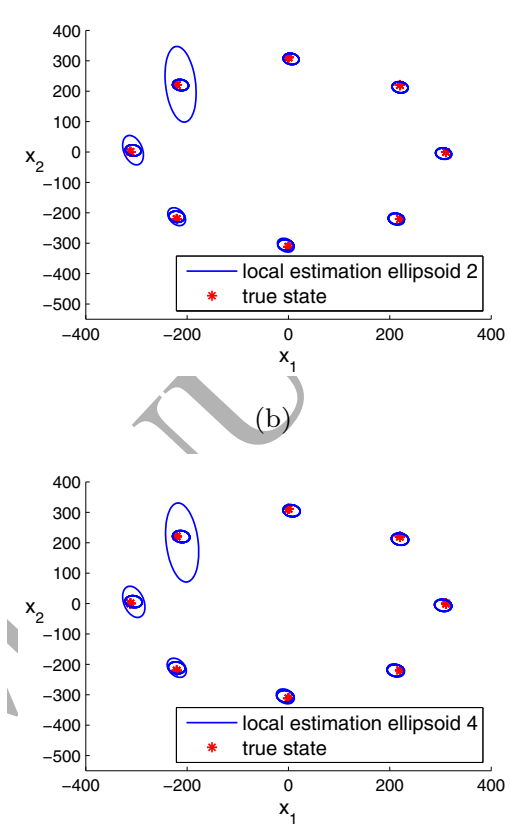

(d)

Figure 5: The ellipsoidal state estimation results of each filter

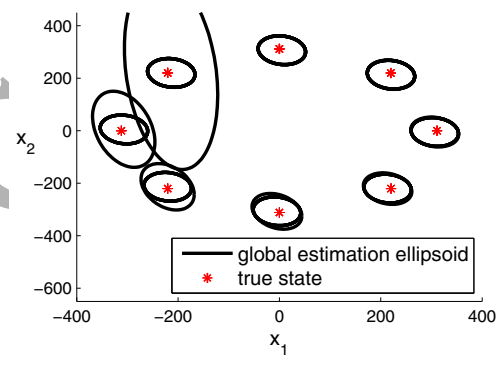

(a)

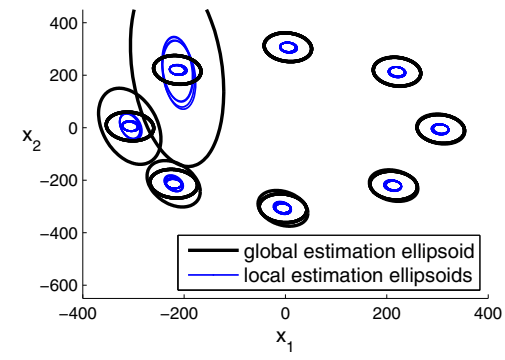

(b)

Figure 6: The global ellipsoidal state estimations 
Remark 8. Note that the experiment is conducted under the unknown-butbounded noises, which is not suitable for performing the DKF approach. Thus, the comparison between the estimation of the DNSMF and that of the DKF cannot be made in this experiment. But one can evidently see that the proposed algorithm is effective and successful under the unknown-but-bounded noises.

\section{Conclusion}

This paper has provided a new approach to deal with the DNSMF problem with ellipsoidal state estimation in the presence of the unknown-but-bounded process and measurement noises. The true state has been located by both local and global ellipsoidal estimations. Moreover, the global estimation can successfully integrate the local estimations by including the union of all the local estimations via the proposed geometric algorithm. The feasibility and effectiveness of the proposed filtering methods have been demonstrated by an illustrative experiment. The presented approach is very useful for target tracking and fault location. The perfect information exchanged among the distributed set-membership filters is considered in this paper. In the future, a challenging research topic will focus on imperfect information among the distributed filter exchange, such as data dropouts, time-delay, and transmission errors.

\section{Acknowledgment}

This work was supported in part by the Australian Research Council Discovery Project under Grant DP160103567.

\section{References}

11] S. Boyd, P. Diaconis, L. Xiao, Fastest mixing Markov chain on a graph, SIAM Review 46 (4) (2004) 667-689.

[2] R. Carli, A. Chiuso, L. Schenato, S. Zampieri, Distributed Kalman filtering based on consensus strategies, IEEE J. Sel. Areas Commun. 26 (4) (2008) 622-633.

[3] F.S. Cattivelli, A.H. Sayed, Diffusion strategies for distributed Kalman filtering and smoothing, IEEE Trans. Autom. Control 55 (9) (2010) 20692084 . 
[4] B. Chen, W.-A. Zhang, L. Yu, Distributed finite-horizon fusion Kalman filtering for bandwidth and energy constrained wireless sensor networks, IEEE Trans. Signal Process. 62 (4) (2014) 797-812.

[5] D. Ding, Z. Wang, B. Shen, H. Dong, Envelope-constrained $H_{\infty}$ filtering with fading measurements and randomly occurring nonlinearities: the finite horizon case, Automatica, 55 (2015) 37-45.

[6] L. Ding, Q.-L. Han, G. Guo, Network-based leader-following consensus for distributed multi-agent systems, Automatica, 49 (7) (2013) $2281 \mathrm{C} 2286$.

[7] H. Dong, Z. Wang, H. Gao, Distributed filtering for a class of time-varying systems over sensor networks with quantization errors and successive packet dropouts, IEEE Trans. Signal Process. 60 (6) (2012) 3164-3173.

[8] H. Dong, Z. Wang, H. Gao, Distributed $H_{\infty}$ filtering for a class of Markovian jump nonlinear time-delay systems over lossy sensor networks, IEEE Trans. Ind. Electron., 60 (10) (2013) 4665-4672.

[9] J. Feng, M. Zeng, Optimal distributed Kalman filtering fusion for a linear dynamic system with cross-correlated noises, Int. J. Syst. Sci. 43 (2) (2012) 385-398.

[10] X. Ge, Q.-L. Han, Distributed event-triggered $H_{\infty}$ filtering over sensor networks with communication delays, Inf. Sci. 291 (2015) 128-142.

[11] X. Ge, Q.-L. Han, Distributed sampled-data asynchronous $H_{\infty}$ filtering of Markovian jump linear systems over sensor networks, Signal Process. 127 (2016) 86-99.

[12] X. Ge, Q.-L. Han, F. Yang, Event-based set-membership leader-following consensus of networked multi-agent systems subject to limited communication resources and unknown-but-bounded noise, IEEE Trans. Ind. Electron., 2016, DOI: 10.1109/TIE.2016.2613929

[13] X. Ge, F. Yang, Q.-L. Han, Distributed networked control systems: A brief overview, Inf. Sci. 380 (2017) 117-131.

[14] G. Guo, L. Ding, Q.-L. Han, A distributed event-triggered transmission strategy for sampled-data consensus of multi-agent systems, Automatica, 50 (5) (2014) 1489C1496.

Distributed networked control systems: A brief overview, Inf. Sci. 380 (2017) 117-131. 
[15] O. Hlinka, F. Hlawatsch, P.M. Djuric, Distributed particle filtering in agent networks: A survey, classification, and comparison, IEEE Signal Process. Mag. 30 (1) (2013) 61-81.

[16] B.J. Kubica, A class of problems that can be solved using interval algorithms, Computing 94 (2-4) (2012) 271-280.

[17] A.B. Kurzhanski, I. Vlyi, Ellipsoidal calculus for estimation and control, Nelson Thornes, 1997.

[18] Q. Lu, Q.-L. Han, B. Zhang, D. Liu, S. Liu, Cooperative control of mobile sensor networks for environmental monitoring: an eventtriggered finite-time control scheme, IEEE Trans. Cybern., 2016, DOI: 10.1109/TCYB.2016.2601110

[19] M.S. Mahmoud, H.M. Khalid, Distributed Kalman filtering: a bibliographic review, IET Control Theory Appli. 7 (4) (2013) 483-501.

[20] I. Matei, J.S. Baras, Consensus-based linear distributed filtering, Automatica 48 (8) (2012) 1776-1782.

[21] A. Ribeiro, G.-B. Giannakis, S.I. Roumeliotis, SOI-KF: Distributed Kalman filtering with low-cost communications using the sign of innovations, IEEE Trans. Signal Process. 54 (12) (2006) 4782-4795.

[22] G.G. Rigatos, Distributed filtering over sensor networks for autonomous navigation of UAV s, Intell. Serv. Robot. 5 (3) (2012) 179-198.

[23] G.G. Rigatos, A derivative-free distributed filtering approach for sensorless control of nonlinear systems, Int. J. Syst. Sci. 43 (9) (2012) 1699-1712..

[24] T. Semertzidis, K. Dimitropoulos, A. Koutsia, N. Grammalidis, Video sensor network for real-time traffic monitoring and surveillance, IET Intell. Transp. Syst. 4 (2) (2010) 103-112.

[25] W. Shang, Y. Kang, H. Xi, Y. Xia, Y.-B. Zhao, Distributed $H_{\infty}$-consensus filtering with sensor networks: a finite horizon solution, IMA J. Math. Control Inf. 31 (1) (2014) 33-49.

[26] S.P. Shary, Solvability of interval linear equations and data analysis under uncertainty, Automation and Remote Control 73 (2) (2012) 310-322. 
[27] H. Song, L. Yu, W.-A. Zhang, Networked $H_{\infty}$ filtering for linear discretetime systems, Inf. Sci. 181 (3) (2011) 686-696.

[28] J. Wang, X.-M. Zhang, Q.-L. Han, Event-triggered generalized dissipativity filtering for neural networks with time-varying delays, IEEE Trans. Neural Netw. Learning Syst. 27 (2016) 77-88.

[29] Y.-L. Wang, Q.-L. Han, Network-based fault detection filter and controller coordinated design for unmanned surface vehicles in network environments, IEEE Trans. Ind. Informat. 12 (5) (2016) 1753-1765.

[30] Y.-L. Wang, Q.-L. Han, Network-based heading control and rudder oscillation reduction for unmanned surface vehicles, IEEE Trans. Contr. Syst. Techn., 2016, DOI: 10.1109/TCST.2016.2617321

[31] G. Wei, S. Liu, Y. Song, Y. Liu, Probability-guaranteed set-membership filtering for systems with incomplete measurements, Automatica 60 (2015) $12-16$.

[32] L. Xiao, S. Boyd, Fast linear iterations for distributed averaging, Syst. Control Lett. 53 (1) (2004) 65-78.

[33] F. Yang, Y. Li, Set-membership filtering for systems with sensor saturation, Automatica 45 (8) (2009) 1896-1902.

[34] F. Yang, Y. Li, Robust set-membership filtering for systems with missing measurement: a linear matrix inequality approach, IET Signal Process. 6 (4) (2012) 341-347.

[35] J. Yoo, W. Kim, H.J. Kim, Distributed estimation using online semisupervised particle filter for mobile sensor networks, IET Control Theory Appl. 9 (3) (2015) 418-427.

[36] H. Yu, Y. Zhuang, W. Wang, Distributed $H_{\infty}$ filtering in sensor networks with randomly occurred missing measurements and communication link failures, Inf. Sci. 222 (2013) 424-438.

[37] H. Zhang, H. Yan, F. Yang, Q. Chen, Distributed average filtering for sensor networks with sensor saturation, IET Control Theory Appl. 7 (6) (2013) 887-893.

[38] X.-M. Zhang, Q.-L. Han, Robust $H_{\infty}$ filtering for a class of uncertain linear systems with time-varying delay, Automatica, 44 (1) (2008) 157C166. 
[40] X.-M. Zhang, Q.-L. Han, Network-based $H_{\infty}$ filtering using a logic jumpinglike trigger, Automatica, 49 (5) (2013) 1428-1435.

[40] X.-M. Zhang, Q.-L. Han, X. Yu, Survey on recent advances in networked control systems, IEEE Trans. Ind. Informat. 12 (5) (2016) 1740-1752.

[41] X.-M. Zhang, Q.-L. Han, B.-L. Zhang, An overview and deep investigation on sampled-data-based event-triggered control and filtering for networked systems, IEEE Trans. Ind. Informat. 13 (1) (2017) 4-16.

[42] S. Zhu, C. Chen, W. Li, B. Yang, X. Guan, Distributed optimal consensus filter for target tracking in heterogeneous sensor networks, IEEE Trans. Cybern. 43 (6) (2013) 1963-1976. 


\section{Accepted Manuscript}

Distributed Networked Set-Membership Filtering with Ellipsoidal State Estimations

Nan Xia, Fuwen Yang, Qing-Long Han

$\begin{array}{ll}\text { PII: } & \text { S0020-0255(17)31130-1 } \\ \text { DOI: } & \text { 10.1016/j.ins.2017.12.010 } \\ \text { Reference: } & \text { INS 13303 }\end{array}$

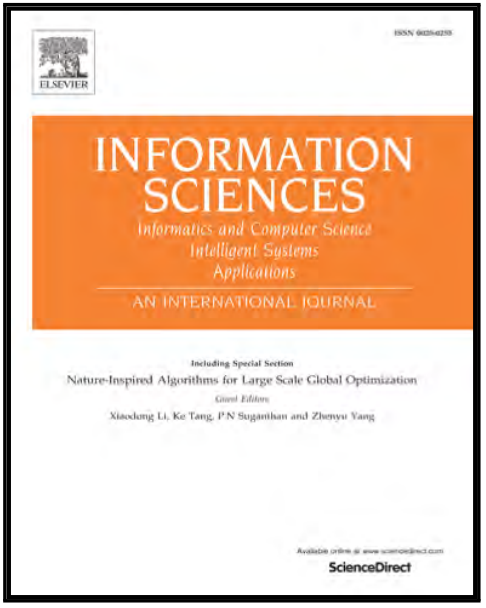

To appear in:

Information Sciences

Received date: $\quad 9$ September 2015

Revised date: $\quad 24$ February 2017

Accepted date: $\quad 6$ December 2017

Please cite this article as: Nan Xia, Fuwen Yang, Qing-Long Han, Distributed Networked SetMembership Filtering with Ellipsoidal State Estimations, Information Sciences (2017), doi: 10.1016/j.ins.2017.12.010

This is a PDF file of an unedited manuscript that has been accepted for publication. As a service to our customers we are providing this early version of the manuscript. The manuscript will undergo copyediting, typesetting, and review of the resulting proof before it is published in its final form. Please note that during the production process errors may be discovered which could affect the content, and all legal disclaimers that apply to the journal pertain. 